\title{
Molecular fluorescence above metallic gratings
}

\author{
Piers Andrew* and William L. Barnes \\ Thin Film Photonics Group, School of Physics, University of Exeter, Exeter, Devon, EX4 4QL, United Kingdom
}

(Received 29 November 2000; published 6 September 2001)

\begin{abstract}
We present measurements of the fluorescence of emitters located in close proximity $(d<\lambda)$ to metallic grating surfaces. By measuring both the spontaneous emission lifetime and angle-dependent radiation pattern of a monolayer of dye molecules as a function of their separation from planar and periodically corrugated mirrors of increasing modulation depth, we are able to examine the effect of varying the surface profile on the emission process. Both the distance dependence of the lifetime and the spatial distribution of the emitted light are significantly changed upon the introduction of a corrugation, quite apart from the appearance of the familiar Bragg-scattered bound-mode features. It is postulated that these perturbations arise from the interference of the grating scattered dipole fields with the usual upward propagating and reflected fields. In addition, the measurement of nonexponential decay transients for the deepest gratings examined provide evidence for the existence of optically dissimilar dipole positions above the grating surface.
\end{abstract}

DOI: 10.1103/PhysRevB.64.125405

PACS number(s): 85.60.Jb, 42.79.Dj, 42.70.Qs, 42.25.Fx

\section{INTRODUCTION}

As noted by Purcell, ${ }^{1}$ the optical properties of an emitter are not intrinsic, but instead depend upon the local photonic mode density (PMD), a direct consequence of Fermi's golden rule. ${ }^{2}$ The process of spontaneous emission $(\mathrm{SpE})$ is thus strongly influenced by the optical environment, since it is this that determines the PMD experienced by the emitter through control of the optical modes to which it can couple. There is tremendous scope to control and/or modify SpE and other processes involving excited species (such as energy transfer or Raman scattering) by embedding the emitter within structures exhibiting restricted geometries, such as microcavities ${ }^{3}$ or photonic crystals. ${ }^{4}$ The confined fields associated with these structures allow control over the PMD sampled by the emitter and so alter both the spectral and spatial distribution of the emitted light along with the $\mathrm{SpE}$ rate of the emitter.

Drexhage et al. ${ }^{5}$ carried out the earliest experimental demonstration of $\mathrm{SpE}$ rate modification by studying the emission from a monolayer of dye molecules close to a metallic mirror. The presence of the mirror in the near field of the molecules was shown to induce changes in both the $\mathrm{SpE}$ rate and the angular distribution of their fluorescence, the degree of modification depending on the exact moleculemirror separation; see Fig. 1. For large separations ( $d$ $>20 \mathrm{~nm}$ ) the $\mathrm{SpE}$ rate was found to display an oscillatory dependence on the dye-mirror separation. This change is induced by the distance-dependent variation in the phase of the retarded molecular dipole fields reflected from the mirror, which drive the dipole. Depending on the phase of these retarded fields, it is possible to observe either enhancement or inhibition of the emission (the dipole is driven in or out of phase, respectively). For molecules in very close proximity to the metallic mirror $(d<20 \mathrm{~nm})$, strong quenching of the emission was observed. This was found to be largely due to the excitation of surface plasmon polaritons (SPP's) propagating at the metal-dielectric interface, as confirmed by Pockrand, Brillante, and Möbius. ${ }^{6}$ The Drexhage results were successfully modelled by Chance, Prock, and Silbey ${ }^{7}$ (CPS) using a classical electrodynamic model that treats the emitter as a forced, damped electric dipole oscillator. This initial, groundbreaking work in the 1970 s led to a plethora of reports studying the emission from excited molecules in restricted geometries. The idea of mode confinement has also been taken into the applied realm; microcavity structures have the potential to enhance the emission from light emitting devices (LED's). ${ }^{8}$ In the main, however, these devices utilize planar interfaces since these are both simple to manufacture and easy to model theoretically.

Perhaps the greatest problem associated with these planar structures is that of light extraction-a large proportion of the emission from sources embedded within the device couples to the bound electromagnetic modes of the structure, such as waveguide modes, and for cavities using metal mirrors, to SPP modes as well. The resulting loss in efficiency has been determined for a variety of structures and can be high. ${ }^{9,10}$ This emission is usually regarded as a loss since it does not escape the structure and thus limits the potential device efficiency; it is a particular problem for devices that have high-refractive-index cavities, such as inorganic semiconductor LED's (Ref. 11) or polymeric devices. ${ }^{12}$

A simple solution is achieved by patterning the interfaces of the device with a periodic wavelength scale corrugation. This microstructure breaks the translational invariance of the interface, allowing the bound modes of the structure to scatter using the Bragg vector associated with the grating corrugation, thereby losing enough momentum to couple to freely propagating radiation. This idea was first demonstrated experimentally for the SPP mode by Knoll et al. ${ }^{13}$ using dye monolayers deposited in a thin film upon a silver grating, shortly followed by Adams et al. ${ }^{14}$ confirming the predictions of Aravind, Hood, and Metiu. ${ }^{15}$ Adams et al. ${ }^{16}$ and Sullivan et al. ${ }^{17}$ have extended the idea by depositing thicker films to study the scattering of waveguide modes. LED (Ref. 18) and quantum well ${ }^{19}$ device structures utilizing grating couplers have recently been fabricated and studied, with a doubling in efficiency observed for the LED device. There is still scope for improvement with optimized device structures; the efficiency with which bound light can be recovered by 
scattering from a corrugation has been shown to be as high as $50 \%$ for the surface plasmon mode, for both single grating $^{20}$ and bigrating ${ }^{21}$ structures. This is significant for LED structures, which necessarily incorporate metal contacts for efficient electron injection.

In all of these studies, the grating has only been considered as a means of facilitating the coupling of the bound modes of the structure (excited by nearby emitters) to freely propagating photons outside the structure. The presence of the grating will, however, also directly affect the SpE of the emitters themselves, since the PMD they experience will be necessarily different from the planar case due to the different boundary conditions. This should lead to changes in both the $\mathrm{SpE}$ rate and the radiation pattern, quite apart from the familiar Bragg-scattered bound-mode reradiation features, which have been the focus of previous studies.

The alteration of the local photonic mode density induced by the grating and the concomitant effect on the SpE rate of the emitter has thus far received little attention, with considerably more theoretical work than actual experiment having been carried out. Initial theoretical studies of the lifetime of dye molecules close to a nonplanar surface were carried out by Arias, Aravind, and Metiu, ${ }^{22}$ who examined the effect of small random surface roughness, and by Leung and co-workers, ${ }^{23}$ who examined shallow periodic grating structures. These last workers only considered the wave vector component of the dipole field normal to the grating $\left(k_{\|}=0\right)$ and predicted order-of-magnitude emission enhancement by gratings of minute amplitude $(\sim 1 \mathrm{~nm})$, due to the excitation of morphology dependent resonances. Since all components of the dipole field need to be considered, even in the case of a planar interface, ${ }^{7}$ it is an open question as to whether the predicted enhancements will be seen experimentally, or whether they are artifacts of the approximation used in the theory. Furthermore, neither of these authors consider the radiation pattern of the emitted light. Recent work by Rigneault et al. ${ }^{24}$ has calculated the field distribution of and power extracted from dipoles within deep grating structures, but at present their theory cannot calculate the power lost within the structure and so cannot give the $\mathrm{SpE}$ rate.

To our knowledge, the only systematic experimental study of $\mathrm{SpE}$ rates has been carried out by Amos and Barnes; ${ }^{25}$ measurements were made of the $\mathrm{SpE}$ lifetime of a monolayer of $\mathrm{Eu}^{3+}$ ions $\left(\lambda_{\mathrm{em}}=614 \mathrm{~nm}\right)$ as a function of the separation from metallic gratings of a variety of pitches. For long pitch gratings with small modulation amplitude (pitch $1075 \mathrm{~nm}$, amplitude $40 \mathrm{~nm}$ and pitch $801 \mathrm{~nm}$, amplitude $19 \mathrm{~nm}$ ), the distance dependence of the lifetime was found to be identical to the planar case. For shorter pitch gratings (pitch $408 \mathrm{~nm}$, amplitude $30 \mathrm{~nm}$ and pitch $408 \mathrm{~nm}$, amplitude $68 \mathrm{~nm}$ ), however, perturbations were seen. Although the range of separations examined was much smaller for these gratings than for the longer pitch gratings, not extending past the first peak in the lifetime-separation curve [Fig. 1(a)], the amplitude of the lifetime oscillation with $\mathrm{Eu}^{3+}$-mirror separation distance appeared to be damped with respect to the planar dependence. Since the oscillation amplitude is primarily dependent on the strength of the reflected field driving the dipole, it was tentatively postulated that scattering of the dipole near field from the grating corrugation diminishes the specularly reflected field at the dipole site. This has the effect of reducing the driving field acting upon the dipole, hence damping the lifetime oscillation with distance. It was concluded that perturbation in the $\mathrm{SpE}$ rate was only observed for grating pitches less than the emission wavelength and that this was because the dipole could not sample the periodicity associated with the longer pitch gratings. If scattering of the dipole near field does occur, it should be observable as a signature in the radiation pattern of the emission from the grating samples. This is because the planar radiation pattern is dependent of the interaction of the upwardly radiated field from the dipole with that reflected from the mirror-if the mirror can also diffract the incident light, then the radiation pattern will depend on the scattered fields too.

In order to see whether such a signature does occur, we have extended the SpE lifetime studies of Drexhage et al. ${ }^{5}$ and Amos and Barnes ${ }^{25}$ by measuring the angle-dependent radiation patterns of a monolayer of emitters in close proximity to a variety of corrugated surfaces in addition to the $\mathrm{SpE}$ lifetime dependence. The fabrication of samples spanning a greatly increased range of $\mathrm{Eu}^{3+}$-ion-mirror separations with respect to earlier work ${ }^{25}$ allows us to confirm that the lifetime perturbation observed previously is indeed a damping of the lifetime oscillation with separation. Furthermore, by studying a series of gratings with increasing modulation amplitude, we observe how this perturbation to the planar SpE lifetime evolves with increase in scattering from the grating corrugation. Deviations from planar behavior observed in the radiation patterns of the deeper grating samples studied suggest that significant scattering of the dipole fields by the corrugation does occur, strengthening the hypothesis forwarded to explain previous deep-grating $\mathrm{SpE}$ lifetime results.

\section{EXPERIMENT}

The mirrors used in this work were formed by the thermal evaporation of a 200-nm-thick silver film onto an appropriately profiled substrate. In the case of planar mirrors, the substrates used were silica sheets of $\lambda / 10$ flatness. Corrugated grating substrates were fabricated in photoresist using a standard interferometric method. A thin film of photoresist (Shipley S1805) was spun onto silica sheets pretreated with HMDS vapor (1,1,1,3,3,3-hexamethyldisilazane) to improve adhesion. (This was important for subsequent LangmuirBlodgett (LB) deposition of monomolecular layers-the surface tension of the floating Langmuir monolayer was found to be sufficiently strong to be able to strip the photoresist away from untreated substrates.) After baking at $100{ }^{\circ} \mathrm{C}$ for 30 min to drive off solvent from the polymer, the grating structures were written into the photoresist as a solubility profile by exposure to an interference pattern formed by the overlap of two coherent beams from an argon ion laser operating at $\lambda=457.9 \mathrm{~nm}$. After development, the resulting surface microstructure was rendered more physically robust by baking in an oven at $150{ }^{\circ} \mathrm{C}$. Using this technique and by varying the exposure time, gratings were made with a pitch, $\lambda_{g}$ of $415 \mathrm{~nm}$, and amplitudes, $a_{0}$ ranging from 20 to 130 
$\mathrm{nm}$. As a precaution, each grating substrate included a planar area surrounding the corrugated region, to act as a direct control. This was necessary because of the possibility of the photoresist surface being different to the silica surface used in the planar mirror experiments (due to different surface roughness, for example) and also to eliminate the possibility of variation between different samples (slightly different silver optical constants). In this way comparison could be made between planar and corrugated mirrors on the same sample, with all other variables being held constant.

Characterization of the grating profiles so produced was achieved by the measurement of surface plasmon resonances in the angle-dependent specular reflectivity of the silvercoated samples. Since the SPPs excited by the incident beam at the metal-dielectric (air) grating interface exhibit a high local field strength, the resonance features apparent in the reflectivity spectra are very sensitive to the interface properties. Fitting theoretical reflectivity data generated using the technique of Chandezon et al. ${ }^{26}$ to these experimental data permitted us to determine the grating profile in the form of a Fourier expansion of harmonic components, as well as the complex dielectric constant of the metal film. ${ }^{27}$

Ten separate regions of differing spacer layer thickness were then deposited onto each grating sample, allowing the study of a number of dye-mirror separations per substrate. The dye-spacer layer system used is similar to that of Drexhage et al. ${ }^{5}$ and Amos and Barnes ${ }^{25}$ and allows us to measure the emission properties of a dye monolayer less than a wavelength distant from the metal surface. A stepped spacer layer structure was built up on top of each mirror by the sequential deposition of monolayers of an optically inert, transparent fatty acid using the Langmuir-Blodgett film deposition technique. The monolayers so transferred follow the grating profile so that the upper surface of the spacer layer structure retains the same profile as the underlying grating. ${ }^{28}$ This was confirmed for a variety of film thicknesses and grating depths by measuring the resultant surface profile after LB film deposition using an atomic force microscope operating in noncontact mode. The material, 22-tricosenoic acid, was deposited at a surface pressure of $30 \mathrm{mN} \mathrm{m}^{-1}$, the speed depending on the substrate used. Film transfer was facile for the planar mirrors and shallow gratings, and a vertical immersion speed of $0.5 \mathrm{~mm} \mathrm{~s}^{-1}$ could be used. For the deeper-grating structures, however, special measures had to be taken and dipping was carried out at $0.05 \mathrm{~mm} \mathrm{~s}^{-1}$ with a 30 -s delay between strokes to allow meniscus draining and prevent surface wetting. The grating grooves and the dipping direction were also kept parallel to ensure minimal contact angle variation. Once the spacer layer structure had been fabricated, a monolayer of the dye material, a $\mathrm{Eu}^{3+}$ complex [N-hexadecylpyridinium tetrakis (1,3-diphenyl-propane-1,3-dionato) europium (III)], was deposited on top using a horizontal transfer method at a surface pressure of $15 \mathrm{mN} \mathrm{m}^{-1}{ }^{29}$ The dye could thus be separated from the metal interface with a precision of $\sim 5.2$ $\mathrm{nm}$ (the bilayer thickness of 22-tricosenoic acid). The $\mathrm{Eu}^{3+}$ ion is an ideal emitter for this kind of study because it has near monochromatic emission at $614 \mathrm{~nm}$ (bandwidth $5 \mathrm{~nm}$ ) arising from an electric dipole transition and also has a long excited-state lifetime $(\sim 1 \mathrm{~ms})$. Furthermore, because of the extremely low absorption cross section at the emission wavelength, the ions are also effectively noninteracting. In the form of a $\mathrm{Eu}^{3+}$ coordination complex, we have the further advantage of easy excitation by UV light (via Förster transfer to the $\mathrm{Eu}^{3+}$ ion from the ligands) and the ability to deposit monolayers using the Langmuir-Blodgett technique.

Initially, the $\mathrm{Eu}^{3+} \mathrm{SpE}$ decay rate was determined by measuring the excited-state lifetime of the ion for each sample region using a time-resolved photon-counting technique. $\mathrm{Eu}^{3+}$ fluorescence was excited using pulsed UV light from a nitrogen laser loosely focused onto the region of interest of the sample $(\lambda=337 \mathrm{~nm}, 5-\mathrm{ns}$ pulses, $30-\mathrm{Hz}$ repetition, $\sim 3-\mathrm{mm}^{2}$ beam spot). The fluorescence was collected using a silica lens, spectrally isolated by a monochromator set to pass $614 \mathrm{~nm}$ (resolution $5 \mathrm{~nm}$ ) and detected using a cooled photomultiplier. Pulse discrimination and arrival time measurement was accomplished using a multichannel photon counter triggered from the laser pulse and a temporal histogram of the SpE decay transient acquired. The SpE lifetime of the $\mathrm{Eu}^{3+}$ was determined for each ion-mirror separation by fitting a multiexponential decay function to the temporal data using a nonlinear least-squares fitting routine, with a $(1 / y)$ statistical weighting. The majority of the data for the planar and corrugated mirror samples were found to be single exponential in form, although a small number of data sets were better fitted by a biexponential model. The deviation from single-exponential behavior, however, was small; the amplitude ratio of the two lifetime components was typically found to be 5:1 with the longer-lifetime component dominant - this is the lifetime that we report below. In all cases, the reduced (i.e., per data point) $\chi^{2}$ value was approximately unity.

To measure the angle-dependent emission from the samples, a slightly different experimental configuration was used. The sample was mounted on a computer-controlled rotating table, allowing the angle made by the sample with respect to the collection optics to be varied. Fluorescence was then excited by UV light directed onto the region of interest from a $600-\mu \mathrm{m}$-core-diameter silica optical fiber comounted on the rotating table. In this way the excitation conditions were kept fixed as the sample was rotated. The table was positioned $200 \mathrm{~mm}$ in front of the monochromator on the axis normal to the collection slits, set at $2 \mathrm{~mm}$ width. Apart from a Glan-Thompson polarizer positioned in front of the entrance slits of the monochromator, no other collection optics were used and so data could be acquired with an angular resolution of $10 \mathrm{mrad}$, which was found to be a suitable compromise between resolution and signal strength. Detection was accomplished in the same manner as for the timeresolved data, but instead of building up a histogram of photon arrival times, the signal was measured in a 1-ms duration "bin," delayed $200 \mu$ s from the trigger pulse and averaged over 200 laser shots. This was repeated for each emission angle and for both TE and TM polarizations; data were typically recorded from $-90^{\circ}$ to $+90^{\circ}$ in $0.5^{\circ}$ steps for each sample region. It was found that the emission intensity fell by about $10 \%$ due to photobleaching during the course of the measurement. Because the bleaching was found to be independent of emission angle, the data could be corrected by 
recording intensities from $-90^{\circ}$ to $90^{\circ}$ and again going from $90^{\circ}$ to $-90^{\circ}$ and averaging the resulting data sets. All radiation patterns were measured in the classical mount; that is to say, the plane of emission sampled was that perpendicular to the grating groove direction.

\section{RESULTS}

\section{A. Planar mirrors}

We first ensured that our experimental system was behaving as expected for a well-studied geometry, that of a planar mirror. $\mathrm{SpE}$ lifetimes were measured for $\mathrm{Eu}^{3+}$-planar mirror separations ranging from 0 to 24022 -tricosenoic acid spacer layers in increments of 4 layers, i.e., from 0 to $650 \mathrm{~nm}$ in 5.2-nm steps. Figure 1(a) shows the experimentally measured $\mathrm{Eu}^{3+} \mathrm{SpE}$ lifetime as a function of the $\mathrm{Eu}^{3+}$-mirror separation for the planar mirror control sample. The solid line is the theoretical dependence obtained from the CPS model, ${ }^{7}$ calculated using the parameters $\varepsilon_{\mathrm{Ag}}=-16+0.6 i$, $\varepsilon_{\mathrm{LB}}=2.49+0.0 i, \quad \lambda_{\mathrm{EM}}=614.7 \mathrm{~nm}, q=0.73 \pm 0.03$, and $\tau_{0}$ $=(0.72 \pm 0.01) \mathrm{ms}$ and assuming an isotropic distribution of dipole moments. Here $\varepsilon_{\mathrm{Ag}}$ and $\varepsilon_{\mathrm{LB}}$ are the optical constants of the silver film and LB spacer layers, respectively, and $\lambda_{\mathrm{EM}}, q$, and $\tau_{0}$ are the emission wavelength, quantum efficiency, and free-space lifetime of the $\mathrm{Eu}^{3+}$ complex. Of these, only $q$ and $\tau_{0}$ were fitting parameters, the other quantities being experimentally determined.

Planar mirror radiation patterns were measured for $\mathrm{Eu}^{3+}$-mirror separations of 20-200 22-tricosenoic acid spacer layers in 20-layer steps. Figures 1(b) and 1(c) show the TE- and TM-polarized radiation patterns measured for an $\mathrm{Eu}^{3+}$-mirror separation of 40 layers $(104 \mathrm{~nm})$, together with the calculated theoretical dependences; similar agreement was found for all other $\mathrm{Eu}^{3+}$-mirror separations measured. The theory patterns were obtained using a simple model that treats the dye layer as an isotropic distribution of independent electric dipoles and considers the interference of their upwardly radiated fields with those reflected from the mirror. ${ }^{5,30}$ The presence of an upper dye layer/air interface means that the effects of multiple beam interference and refraction also have to be considered. Again, the parameters used in the model were the optical constants of the silver mirror and spacer layer, both of which were obtained from independent measurements, and the $\mathrm{Eu}^{3+}$-mirror separation. The excellent agreement between experiment and theory for the planar mirror system in both the SpE lifetime and radiation pattern measurements demonstrated above gives us confidence in our experimental technique and allows us to examine the effects of mirror corrugation.

\section{B. Long-pitch gratings}

A structure with minimal perturbation from a planar interface was then studied, that of a shallow long-pitch grating (pitch $800 \mathrm{~nm}$ and amplitude $10 \mathrm{~nm}$ ). The dependence of the $\mathrm{SpE}$ lifetime on the $\mathrm{Eu}^{3+}$-mirror separation measured for this sample is shown in Fig. 2(a), together with the planar mirror theory; it can be seen that there is little difference between them, as may be expected for such a small perturbation. ${ }^{25}$
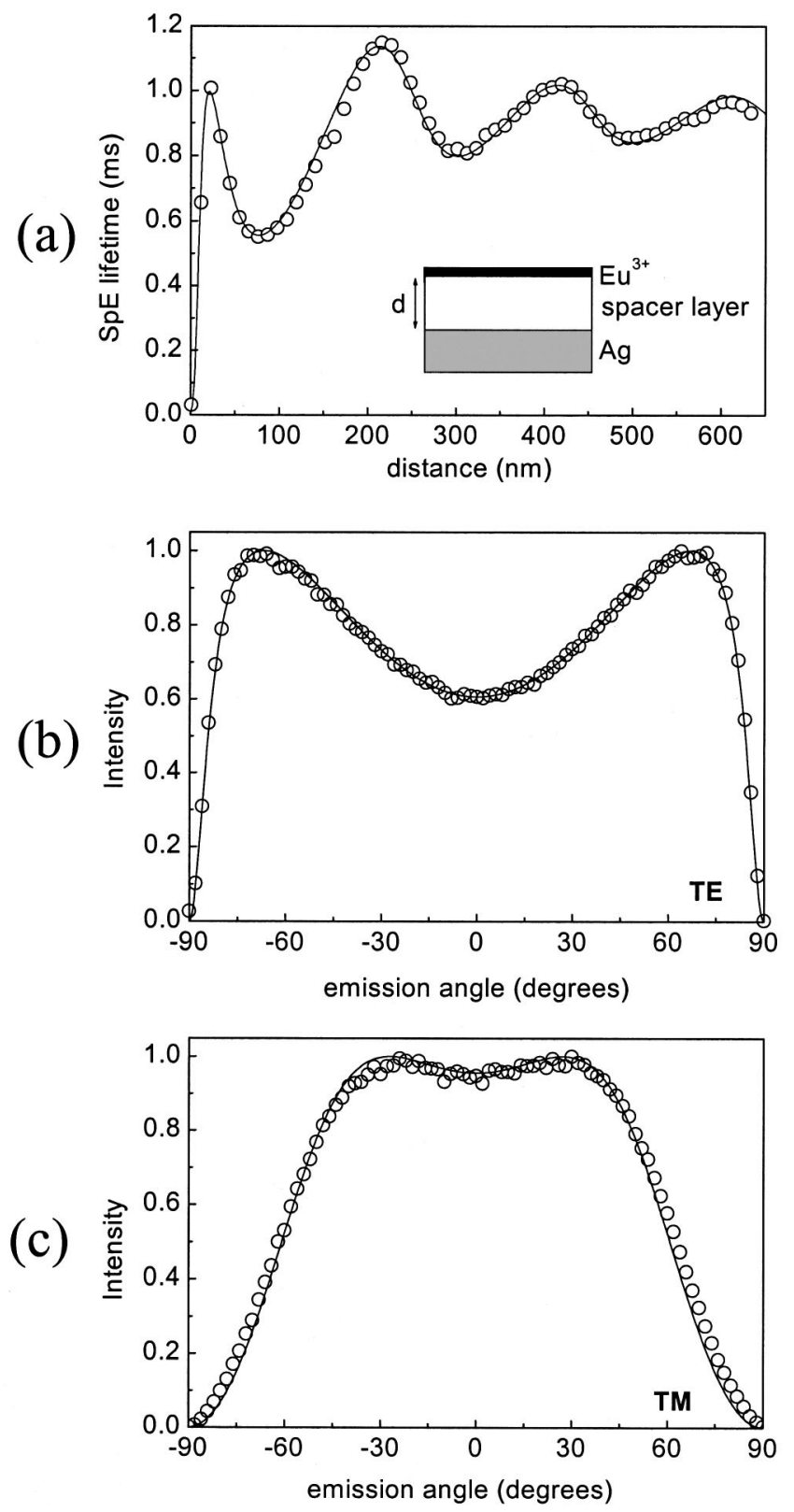

FIG. 1. SpE lifetime of a monolayer of $\mathrm{Eu}^{3+}$ ions situated above a planar silver mirror as a function of ion-mirror separation (a) and the angle-dependent radiation patterns for TE- and TM-polarized emission with an ion-mirror separation of $104 \mathrm{~nm}$ (b), (c). In each case, the open circles are the experimental data, while the solid line is the theoretical model as described in the text. The inset in (a) shows the experimental geometry.

TE- and TM-polarized radiation patterns measured for the sample region with a $\mathrm{Eu}^{3+}$-mirror separation of 60 layers $(156 \mathrm{~nm})$ are depicted in Figs. 2(b) and 2(c), together with the calculated planar radiation patterns for an $\mathrm{Eu}^{3+}$-mirror separation of $156 \mathrm{~nm}$. Because of the presence of a corrugated interface, there are now two contributions to the radiation pattern; the slowly varying background emission familiar from the planar sample is superposed with intense narrow emission peaks. These peaks correspond to the gratingcoupled reradiation from bound modes excited by the $\mathrm{Eu}^{3+}$ 
(a)

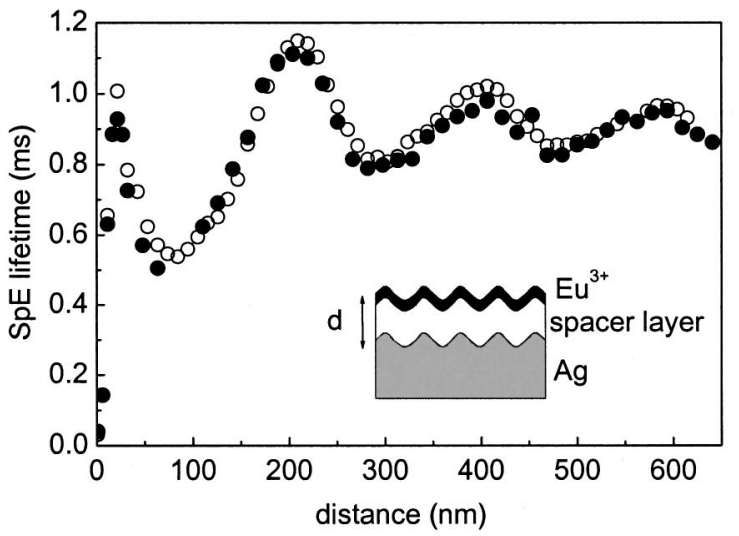

(b)

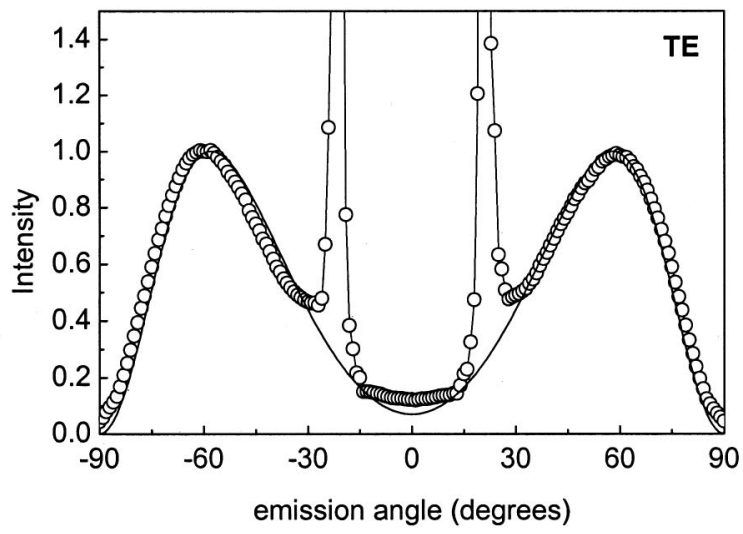

(c)



FIG. 2. SpE lifetime of a monolayer of $\mathrm{Eu}^{3+}$ ions situated above a silver grating of pitch $800 \mathrm{~nm}$ and amplitude $10 \mathrm{~nm}$ as a function of ion-mirror separation (a) and the angle-dependent radiation patterns for TE- and TM-polarized emission with an ion-grating separation of $156 \mathrm{~nm}$ (b), (c). For (a), the solid circles are the grating data, while the open circles are those for a planar mirror. For (b) and (c), the open circles are the experimental grating data and the solid line is the planar theoretical model as described in the text. Again, the inset in (a) shows the experimental geometry.

monolayer. For the remainder of this paper, we denote the slowly varying background as the direct dipole emission and the grating-scattered bound-mode peaks as the indirect emission. It can be seen that the direct dipole emission component of the grating radiation pattern is well modeled by the planar theory, so that the only perturbations induced by the grating are the scattered mode features. In the TE-polarized radiation pattern the sharp emission peak at $\sim 21^{\circ}$ corre- sponds to the scattered $\mathrm{TE}_{0}$ waveguide mode; the TMpolarized pattern displays the reradiation of the $\mathrm{TM}_{0}$ mode (SPP) at $\sim 60^{\circ}$ in addition. Weak scattered reradiation from the $\mathrm{TE}_{0}$ mode appears in the TM-polarized radiation pattern because the slight optical anisotropy of the 22-tricosenoic acid guide layer gives the modes a mixed polarization state. ${ }^{31}$ Measurements made for greater $\mathrm{Eu}^{3+}$-mirror separations reveal radiation patterns of similar form to those of Figs. 2(b) and 2(c). In each case, the characteristic planar direct dipole emission is superposed with the indirect reradiation from scattered guided modes, more in number since the thicker guiding (spacer) layer can support higher order waveguide modes. By measuring the angles at which these modes are reradiated as a function of guide thickness, we can reconstruct the mode dispersion curve for the structure, in good agreement with theory. ${ }^{16,32}$

In summary, save for the appearance of scattered boundmode features, the radiation patterns of $\mathrm{a} \mathrm{Eu}^{3+}$ monolayer above a shallow grating are identical to those of a planar structure with equivalent dye-mirror separation. Because the radiation pattern is determined by the interaction of the upwardly radiated dipole fields with those reflected or scattered from the interface, this suggests that the dipole near field does not scatter from the grating and thus does not sample the grating corrugation. This supports the observation that the presence of the corrugation does not affect the dye SpE lifetime distance dependence. The reason that guided modes excited by the dye layer can scatter from the grating while the dipole field itself does not is that the guided modes have a propagation distance greatly in excess of the grating pitch and therefore sample many grating periods, unlike the dipole near field, which extends roughly a wavelength from the source.

\section{Short-pitch gratings}

To see if the dipole near field does sample and can thus scatter from surface corrugation, we studied the $\mathrm{SpE}$ from samples with a greater perturbation from planarity, the shorter-pitch grating samples. Figures 3(a)-3(d) show the $\mathrm{SpE}$ lifetime distance dependences measured for $\mathrm{Eu}^{3+}$ monolayers located above gratings with pitch $415 \mathrm{~nm}$ and amplitudes of (a) $20 \mathrm{~nm}$, (b) $40 \mathrm{~nm}$, (c) $75 \mathrm{~nm}$, and (d) $130 \mathrm{~nm}$, respectively. In each case, the distance dependences measured for the planar regions of the samples are included for comparison, together with the expected planar theory dependence calculated using experimentally determined parameters. For the shallowest grating (which is thus least perturbed from planarity), there is little difference between the grating and control regions, but as the grating amplitudeand hence scattering efficiency-increases, the discrepancy becomes more obvious. In particular, the feature comprising the trough at 28 spacer layers $(72.8 \mathrm{~nm})$ and the peak at 80 spacer layers $(208 \mathrm{~nm})$ begins to flatten out with increasing amplitude, hinting that the characteristic lifetime oscillation with dye-mirror separation is being washed out, in agreement with previous studies. ${ }^{25}$ For the deepest-grating structure, with amplitude $130 \mathrm{~nm}$, the oscillation has been completely flattened, suggesting that the dipole near-field interference 
(a)



(c)

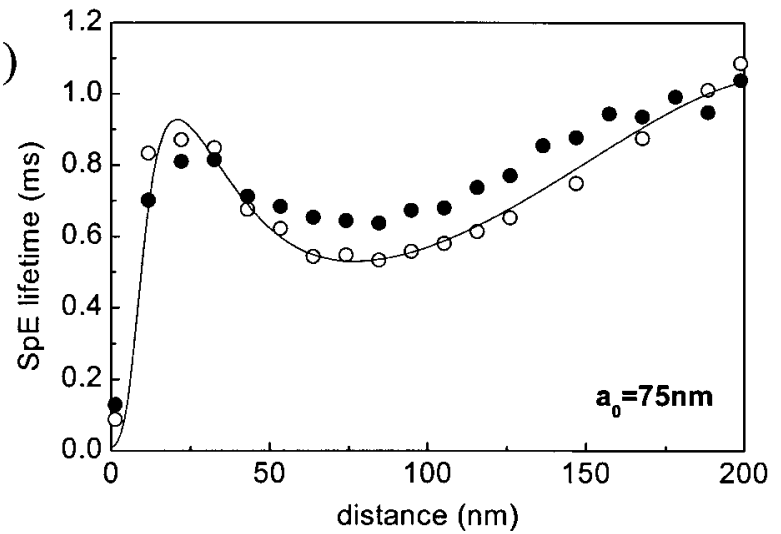

(b)

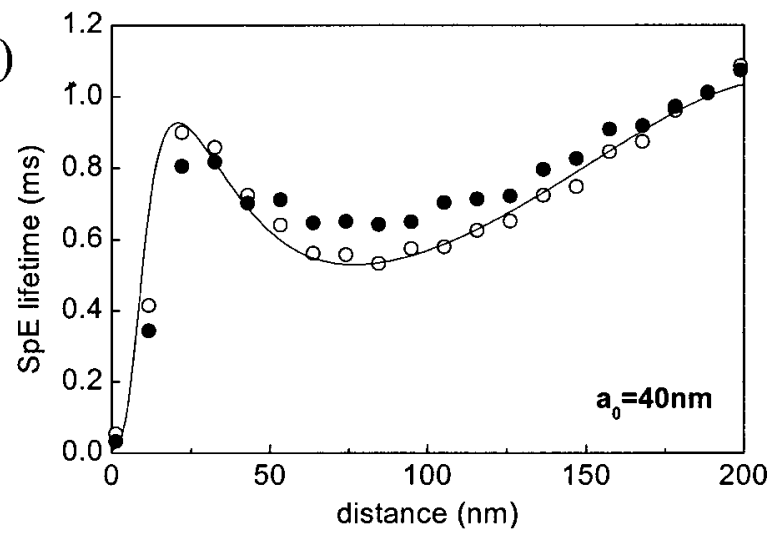

(d)

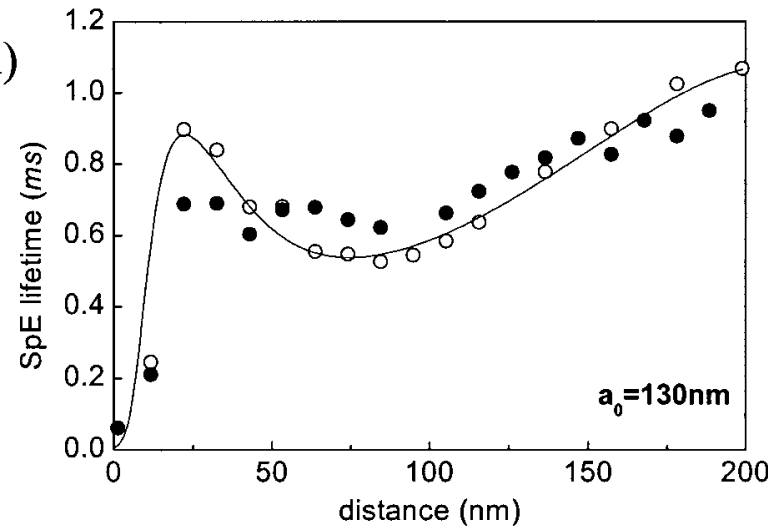

FIG. 3. SpE lifetime of a monolayer of $\mathrm{Eu}^{3+}$ ions situated above silver gratings of pitch $415 \mathrm{~nm}$ and amplitudes of (a) $20 \mathrm{~nm}$, (b) $40 \mathrm{~nm}$, (c) $75 \mathrm{~nm}$, and (d) $130 \mathrm{~nm}$ as a function of ion-grating separation. In each case, the solid circles are the grating data, the open circles are the planar control data, and the solid line is the planar theoretical model. As the grating amplitude increases, a discrepancy between the planar and grating dependencies becomes apparent.

condition has been radically altered and that the dipole near field is indeed strongly scattered from the deeper gratings. To test this hypothesis, the SpE lifetime distance dependence was measured over a greater range of $\mathrm{Eu}^{3+}$-mirror separations to include more periods in the lifetime oscillation. These data are presented in Figs. 4(a) and 4(b) for gratings with a pitch of $415 \mathrm{~nm}$ and amplitudes 30 and $68 \mathrm{~nm}$, respectively, together with the appropriate planar mirror data. The reduction in the amplitude of the oscillation is particularly clear, and the 68 -nm grating amplitude results are more damped than the $30-\mathrm{nm}$ results. This confirms that as the interface is perturbed from planarity, the $\mathrm{SpE}$ lifetime of the $\mathrm{Eu}^{3+}$ ions-and hence the PMD they experience-changes markedly. Figures 3 and 4 show that the SpE lifetime for small $\mathrm{Eu}^{3+}$-mirror separations decreases as the grating amplitude increases. This small separation quenching is primarily due to the excitation of SPP modes at the metal-LB spacer interface by the $\mathrm{Eu}^{3+}$ ions. The increased quenching implies that the coupling to and the nature of the SPP modes propagating at the surface are significantly perturbed by the grating corrugation, a point we return to later in connection with the observed radiation patterns.

As we noted earlier, the bulk of the grating SpE lifetime data was well fitted by a single-exponential decay function (or a slight perturbation from one) and so no difference could be detected in the $\mathrm{SpE}$ from $\mathrm{Eu}^{3+}$ ions occupying different lateral sites with respect to the grating profile. In contrast, the SpE lifetime data for the deepest grating studied (pitch 415 $\mathrm{nm}$, amplitude $130 \mathrm{~nm}$ ) were significantly nonexponential, implying that different $\mathrm{Eu}^{3+}$ ions in the monolayer do experience different local optical environments for this structure. This behavior is illustrated by the $\mathrm{SpE}$ decay data for the 32 -spacer-layer region $(83 \mathrm{~nm})$ of the $130-\mathrm{nm}$ grating, given in Fig. 5 for the (a) planar and (b) corrugated regions of the sample, together with the deviation from a singleexponential fit. While the single-exponential function models the planar data well, significant discrepancies can be seen for the grating data. Without knowledge of the precise deexcitation routes available, it is difficult to determine the appropriate decay function for nonexponential decay data, and so a simple biexponential model was used to approximate the deviation. ${ }^{33}$ In this case, fitting resulted in two lifetime components of roughly equal amplitude, the short component being independent of the ion-mirror separation; it is the longer-lifetime component that is presented in Fig. 3(d).

In common with the shallow grating results, the radiation patterns measured for these deeper-grating samples were composed of two distinct contributions: the direct emission resulting from the interference of the dipole fields, superposed with the indirect scattered bound mode reradiation. Unlike the shallow-grating results, however, the direct dipole emission background contribution displayed significant de- 



FIG. 4. SpE lifetime of a monolayer of $\mathrm{Eu}^{3+}$ ions situated above silver gratings of pitch $415 \mathrm{~nm}$ and amplitudes of (a) $30 \mathrm{~nm}$ and (b) $68 \mathrm{~nm}$ as a function of ion-mirror separation. The solid circles are the grating data, while the open circles are the planar control data. The extended range of ion-grating separations considered shows the reduction in the amplitude of the lifetime oscillation with grating amplitude.

viations from the equivalent $\mathrm{Eu}^{3+}$-mirror separation planar radiation pattern. The discrepancy was most pronounced for $\mathrm{Eu}^{3+}$-mirror separations for which the $\mathrm{SpE}$ lifetime displayed the greatest difference from the planar mirror value. Figures 6(a) and 6(b) compare the TE- and TM-polarized radiation patterns for the 104-spacer-layer $(270 \mathrm{~nm})$ region of the 415-nm-pitch, 30-nm-amplitude grating with the planar theory, while Figs. 6(c) and 6(d) show the corresponding data for the 415-nm-pitch, 68-nm-amplitude grating. This particular spacer layer thickness corresponds to the second minima in the separation dependence of the lifetime, where both samples exhibited the greatest lifetime difference between the planar and grating regions (see Fig. 4). It was thus expected that any change in the radiation pattern caused by the scattering of the dipole field would be most obvious for this spacer layer thickness. Figure 6 shows that the angular dependence of the direct emission from the $\mathrm{Eu}^{3+}$ monolayer is significantly different from the results expected for a planar sample of the same thickness, for both short-pitch gratings considered. Instead of there being a maximum emission normal to the substrate as expected for a planar mirror, the 30-nm-amplitude grating exhibits two radiation lobes at approximately $50^{\circ}$, while the 68 -nm-amplitude grating does exhibit maximum normal emission, but with a different angle dependence. This change in angle dependence for the direct dipole emission with respect to the planar radiation pattern suggests that the interference condition for the dipole near field has been significantly altered by the presence of the corrugation of the deeper-grating samples. In this case, therefore, it appears that the corrugation does scatter the dipole fields, in agreement with the supposition used to explain the damping of the oscillations in the mirror-separationdependent $\mathrm{Eu}^{3+}$ lifetime measured for these samples. To account for the observed changes in the radiation patterns by simply assuming that the grating samples have a different $\mathrm{Eu}^{3+}$-mirror separation to that expected requires an increase in the separation of about 20 monolayers $(52 \mathrm{~nm})$, which is unrealistic; the discrepancy really does arise from the grating corrugation. It is interesting to note that these radiation patterns (for gratings differing only in modulation amplitude) themselves display different direct dipole emission, implying that the dipole near field is affected differently by the microstructure in each case.

The grating corrugation not only affects the direct emission, but also alters the SPP mode and its reradiation, as can be seen in Fig. 7. In this figure, the TM-polarized radiation patterns for the four-spacer-layer region are presented for the 30-, 68-, 75-, and 130-nm-amplitude gratings. The patterns have been normalized so that the background emission peaks at unity. The main feature observable for each radiation pattern is the SPP reradiation peak, which becomes progressively more damped as the amplitude of the grating corrugation increases. The intensity of the emission decreases (with respect to the direct emission), and at the same time the emission broadens and shifts to higher angle, again suggesting that the SPP mode giving rise to the emission is being severely perturbed by the grating corrugation. This is associated with the increased $\mathrm{SpE}$ lifetime quenching observed in this $\mathrm{Eu}^{3+}$-mirror separation regime (Figs. 3 and 4).

\section{DISCUSSION}

The fluorescence decay measurements presented here show that the SpE rate of dye molecules positioned above the shorter-pitch gratings was significantly altered by the grating corrugation, in contrast with the results obtained from a long-pitch grating sample. For the shallowest gratings examined (amplitude $20 \mathrm{~nm}$ ), the lifetime separation dependence was unchanged from that of a planar surface, suggesting that the interface still appeared flat to the dye molecules. As the grating amplitude was increased, the magnitude of the lifetime oscillations for larger $\mathrm{Eu}^{3+}$-mirror separations decreased significantly. The oscillation in the lifetime of the $\mathrm{Eu}^{3+}$ ions for a planar mirror is caused by the separationdependent variation in the retardation of the reflected field driving the dipole. We attribute the reduction in amplitude of this oscillation for corrugated mirrors to the grating scattering of the dipole near field. This will change the driving field at the dipole site by both decreasing the amplitude of the specularly reflected field, as well as generating scattered fields that will interfere with the reflected field.

The proposal that the change in $\mathrm{SpE}$ rate was caused by the scattering of the dipole fields for the deeper grating struc- 



FIG. 5. SpE decay transients measured for a monolayer of $\mathrm{Eu}^{3+}$ ions positioned $83 \mathrm{~nm}$ above (a) a planar silver control region and (b) a silver grating of pitch $415 \mathrm{~nm}$ and amplitude of $130 \mathrm{~nm}$ fabricated on the same substrate. In each case, the lower panel shows the single-exponential fit (solid line) to the experimental data (open circles) obtained using a nonlinear least-squares fitting routine, while the upper panel shows the deviation of this fit from the experimental data $\left(I_{\text {expt }}-I_{\text {fit }}\right)$. The reduced $\chi^{2}$ value for the planar fit is 0.5 , while for the grating fit it is 5.2 .

tures studied is substantiated by the radiation pattern measurements subsequently made. Deviations from the expected planar angle dependence of the direct radiation from the dye monolayer were found for grating samples exhibiting decreased lifetime oscillations. Since the form of the planar radiation pattern is determined by the interference of the dipole fields emitted upwardly with those reflected from the interface, any discrepancy suggests that the interference conditions have been altered. This is likely to be the case if there is substantial scattering of the dipole fields by the grating corrugation.

The scattering is expected to be more important for the shorter-pitch gratings considered here since the dipole near fields are able to sample more of the surface corrugation. The scattering will also be greater because of the large grating amplitudes studied for these shorter-pitch structures. Because of this, it is uncertain whether pitch is a significant factor in determining the degree of scatter for a given wavelength emitter. A parameter allowing comparison of the diffraction efficiency of gratings with differing pitches is the normalized depth or depth-to-pitch ratio $\left(d / \lambda_{g}\right) .{ }^{34}$ Significant changes in the spontaneous emission rate of the dye molecules above the 415-nm-pitch gratings were only observed for amplitudes $30 \mathrm{~nm}$ and greater, corresponding to depth-to-pitch ratios in excess of 0.14 , tallying well with the results of a previous study. $^{25}$ In that study, the aspect ratio of the shorter-pitch gratings was considerably greater than that of the longerpitch gratings, the depth-to-pitch ratio being 0.072 and 0.048 for the 800-nm-pitch gratings and 0.15 and 0.3 for the 400 $\mathrm{nm}$-pitch gratings. In order to confirm that gratings with pitch greater than the emission wavelength do not strongly scatter the dipole fields, it will be necessary to study longpitch gratings with commensurate scattering efficiencies to those short-pitch structures studied here, i.e., 800-nm pitch with amplitude $40 \mathrm{~nm}$.

The importance of grating scattering can be appreciated by consideration of the angle-dependent reflectivity of the grating structures used. The reflectivity of the shallowest grating (amplitude $20 \mathrm{~nm}$ ) is high, only falling below $80 \%$ for a very narrow angle range $\left(\sim 2^{\circ}\right)$ when the SPP mode is excited. The reflectivity of the deeper grating (amplitude 75 $\mathrm{nm}$ ), by way of contrast, is only $\sim 5 \%$ for incident angles above the critical edge at $30^{\circ}$. This is due to the strong diffraction that scatters incident light into to the -1 order for this grating structure. If the dipole fields are able to sample this corrugation, similarly strong scattering can occur, leading to a reduction in the intensity of the specularly reflected field at the dipole site. Since it is the reflected fields that affect the dipole emission rate, any decrease in these fields will cause smaller changes in the SpE lifetime and thus emis- 
(a)

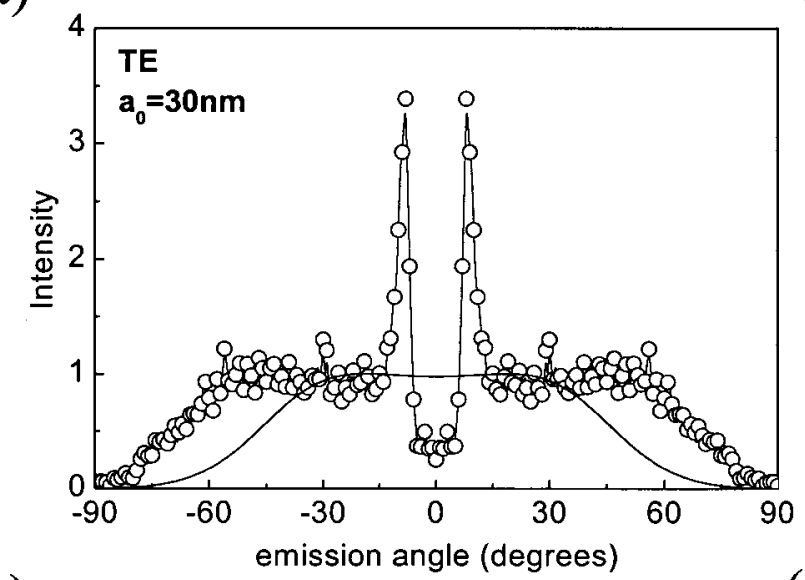

(b)



(c)

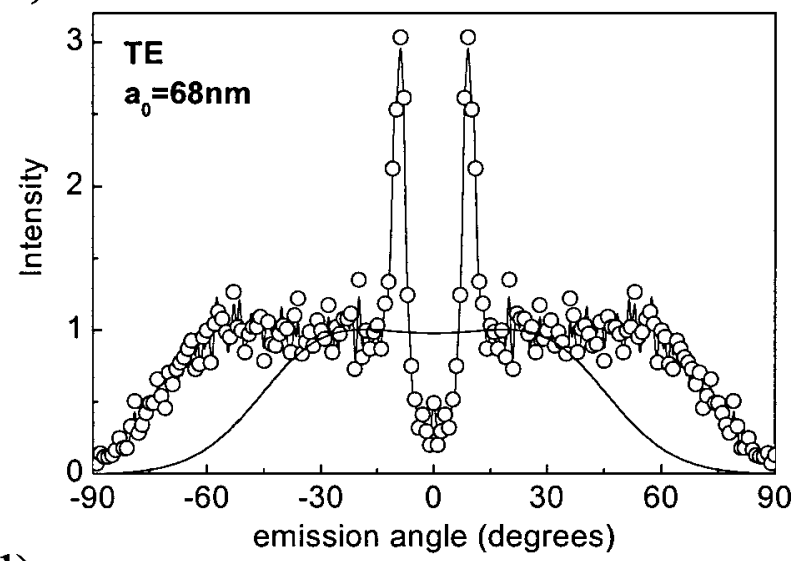

(d)



FIG. 6. Angle-dependent radiation patterns measured for a monolayer of $\mathrm{Eu}^{3+}$ ions positioned $270 \mathrm{~nm}$ above silver gratings with a pitch of $415 \mathrm{~nm}$. Data for the 30-nm-amplitude grating are given in (a) for TE-polarized and (b) TM-polarized emission, while the corresponding data for the 68-nm-amplitude grating is given in (c) for TE-polarized and (d) TM-polarized emission. The open circles are the experimental data, while the solid line is the planar theory calculated for the same ion-mirror separation.

sion rate. The influence of the scattered orders also needs to taken into account, including their phase relative to the specular field, since these will interfere with the specular field, thus affecting the resultant field driving the dipole.

The increased quenching of the $\mathrm{SpE}$ lifetime for small $\mathrm{Eu}^{3+}$-mirror separations for the deeper gratings is caused by perturbation of the SPP modes by the grating corrugation. It is known that the wave vector of the SPP mode propagating at a corrugated interface increases with the grating amplitude; ${ }^{35}$ the associated broadening leads to a greater density of SPP modes per unit wave vector for a given frequency. An increased density of states at the dye emission frequency allows the molecules to couple more strongly to the SPP mode of the metal grating surface, increasing their emission rate. The change in nature of the SPP modes with increasing grating amplitude was confirmed by the radiation pattern measurements showing that the mode was substantially damped and broadened for the deeper grating structures. The exact effect on the lifetime is difficult to predict without a appropriate theoretical model, since the molecule can excite SPP's propagating at any azimuthal angle at the grating interface, and the grating only significantly perturbs the SPP's propagating parallel to the Bragg vector. In at- tempting to explain the lifetime dependence with distance from a metallic grating, we must also consider the effect of energy gaps in the SPP dispersion. ${ }^{36}$ If the grating has an energy gap for the propagation of SPP modes at the emission frequency of the dye, then the quenching of the dye by excitation of SPP modes will be severely reduced. This will lead to changes in the lifetime dependence for small $\mathrm{Eu}^{3+}$-mirror separations, where coupling to the SPP mode is most important. For a single grating corrugation such as that considered here, the gap does not extend over all azimuthal angles for SPP propagation on the surface, but only extends over a limited angle range determined by the grating pitch/ wavelength ratio and the gap width. ${ }^{36}$ Dye molecules can excite SPP's propagating in all azimuthal directions on the metal surface, and so the effect of the gap is clearly limited by the angle range over which it can restrict the propagation of SPP's. The increase of gap width with grating amplitude leads to a greater azimuthal extent of the SPP energy gap. ${ }^{36}$ This implies that the deeper grating results should be influenced more by the existence of an SPP gap and should exhibit reduced molecule-SPP coupling, as noted by Amos and Barnes. ${ }^{25}$

This contradicts the experimental evidence presented ear- 


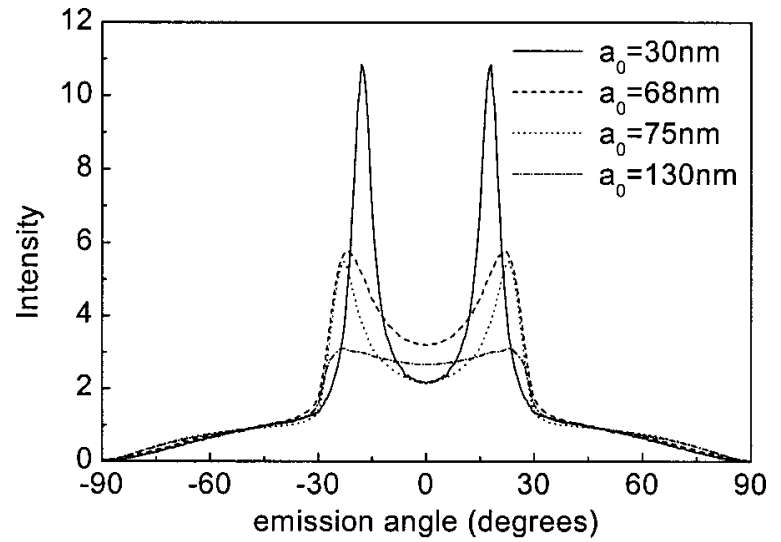

FIG. 7. Angle-dependent radiation patterns measured for TMpolarized emission from a monolayer of $\mathrm{Eu}^{3+}$ ions positioned 10.4 $\mathrm{nm}$ above silver gratings of pitch $415 \mathrm{~nm}$ and amplitudes ranging from 30 to $130 \mathrm{~nm}$.

lier; the quenching of the molecules in the limit of small $\mathrm{Eu}^{3+}$-mirror separations is increased for the larger amplitude gratings. The existence of the SPP gap, however, also implies the existence of band edges, which as standing waves have an increased density of states per unit frequency interval. The molecule-SPP coupling will thus be increased for the band edges, in direct competition with the effect of the gap. Which process is dominant in determining the extent of molecular quenching is hard to judge in the absence of a rigorous theoretical model.

The fluorescence decays measured were single exponential for all but the deepest structure considered. This suggests that there is no difference in the excited-state lifetime for molecules located above the grating peaks and troughs for these structures. The deepest grating studied, of amplitude $130 \mathrm{~nm}$, did exhibit nonexponential fluorescence decays, indicating that there was a difference in the excited-state lifetime for molecules laterally displaced with respect to the grating profile. As the ion-metal separation increased for this sample, the deviation from a single-exponential decay decreased, ${ }^{32}$ perhaps indicating the increasing equivalence of the different lateral dipole sites. Even though there is a distribution of different lifetimes for molecules laterally displaced across a grating groove, it will be hard to resolve any change from single-exponential behavior if the perturbation from planarity is small and the extreme lifetime components are similar in value. The nonexponential behavior of fluorescence decays will be discernible only if the extreme lifetime components are sufficiently different in value, which explains why nonexponentiality was seen only for the deepest structure.

Fluorescence was collected from an ensemble of emitters in these experiments, so a further consideration is whether this ensemble was excited uniformly; i.e., were the $\mathrm{Eu}^{3+}$ ions excited equally irrespective of lateral position with respect to the grating profile? Using the technique of Chandezon et al. ${ }^{26}$ the intensity of the excitation field experienced by the emitters was calculated as a function of lateral position across the grating. ${ }^{37}$ For the structures used in this study, it was found that this excitation profile varied in a nontrivial fashion with both grating depth and overlayer thickness. To find out what overall effect this nonuniform excitation has requires knowledge of the dipole emission characteristics (rate/radiation pattern) as a function of position across the grating profile. As yet, no theory is able to predict this for our system, highlighting the need for further theoretical and experimental investigation.

To overcome this lateral emission variation, a siteselective excitation and/or collection technique is required. One way to accomplish this may be to excite the emitters using an interference pattern with the same pitch as the grating and to translate this excitation profile with respect to the sample. Another possible method exploits the periodicity of the band-edge modes of a SPP band gap. The low- and highenergy modes have field maxima located at peaks and troughs of the grating profile, respectively, and so excitation of the molecules by coupling light into these modes will allow preferential excitation of molecules located at the extrema of the grating profile. This technique can also be applied to the selective collection of light emitted by the molecules, since they will preferentially couple to different SPP modes depending on their lateral position. Alternatively, one could use a near-field scanning optical microscope to selectively excite and collect emission from the sample, although care must be taken in interpreting the resulting information, since the presence of the scanning tip perturbs the system. ${ }^{38}$ This approach has recently been used to study the modification of the emission from a single molecule by the proximity of a nanometer-sized object ${ }^{39}$ indicating the potential of the technique.

The use of corrugation to allow "band engineering" has great potential to modify $\mathrm{SpE}$ rates and emission efficiency. As well as introducing band gaps into mode propagation, reducing the $\mathrm{PMD}$ and thus inhibiting $\mathrm{SpE}$, it should also be possible to use the enhanced PMD at band edges to significantly enhance the SpE rate and, hence, emission intensity. Extending this idea, extremely flat bands with correspondingly large PMD can be generated using asymmetrically profiled waveguides. ${ }^{28}$ It should be possible for dipoles to strongly couple to these modes, with consequent large effect on the $\mathrm{SpE}$ rate. In the absence of experimental measurements, however, it is uncertain as to whether this will actually be achieved.

\section{CONCLUSIONS}

We have studied the fluorescence emission from a monolayer of $\mathrm{Eu}^{3+}$ ions above silver grating structures possessing a variety of pitches and amplitudes in order to determine the effect of the surface corrugation on the SpE process. The distance dependence of the spontaneous emission rate of the $\mathrm{Eu}^{3+}$ ions was shown to change little from the planar dependence for the shallow-grating structures considered, but significant changes were observed for the deeper-grating structures (those with depth-to-pitch ratios greater than 0.1). The radiation patterns of the emission from the grating structures were also measured and consisted of direct emission from the dye molecules and reradiated emission from surface and 
waveguide modes scattered by the corrugation. While direct emission from the shallow gratings resembled that from planar mirror, the direct emission from the deeper structures did not, indicating that the dipole fields were scattered by the deeper corrugations.

All fluorescence decays observed were of singleexponential form, except those for the deepest-grating structure studied. The nonexponential decays measured for this

*FAX: 441392 264111. Electronic address: pandrew@exeter.ac.uk

${ }^{1}$ E. M. Purcell, Phys. Rev. 69, 681 (1946).

${ }^{2}$ E. Fermi, Rev. Mod. Phys. 4, 87 (1932).

${ }^{3}$ R. E. Slusher and C. Weisbuch, Solid State Commun. 92, 149 (1994).

${ }^{4}$ J. Joannopoulos, R. Meade, and J. Winn, Photonic Crystals (Princeton University Press, Princeton, 1995).

${ }^{5}$ K. H. Drexhage, M. Fleck, H. Kuhn, F. P. Schäfer, and W. Sperling, Ber. Bunsenges. Phys. Chem. 70, 1179 (1966); K. H. Drexhage, H. Kuhn, and F. P. Schäfer, ibid. 72, 329 (1968); K. H. Drexhage and M. Fleck, ibid. 72, 330 (1968); K. H. Drexhage, in Progress in Optics, edited by E. Wolf (North-Holland, Amsterdam, 1974), Vol. XII, p. 165.

${ }^{6}$ I. Pockrand, A. Brillante, and D. Möbius, Chem. Phys. Lett. 69, 499 (1980).

${ }^{7}$ R. R. Chance, A. Prock, and R. Silbey, Adv. Chem. Phys. 37, 1 (1978).

${ }^{8}$ H. Benisty, H. de Neve, and C. Weisbuch, IEEE J. Quantum Electron. 34, 1632 (1998).

${ }^{9}$ H. Rigneault, S. Robert, C. Begon, B. Jacquier, and P. Moretti, Phys. Rev. A 55, 1497 (1997).

${ }^{10}$ P. T. Worthing, R. M. Amos, and W. L. Barnes, Phys. Rev. A 59, 865 (1999).

${ }^{11}$ I. Schnitzer, E. Yablonovitch, C. Caneau, T. J. Gmitter, and A. Scherer, Appl. Phys. Lett. 63, 2174 (1993).

${ }^{12}$ N. C. Greenham, R. H. Friend, and D. D. C. Bradley, Adv. Mater. 6, 491 (1994).

${ }^{13}$ W. Knoll, M. R. Philpott, and J. D. Swalen, J. Chem. Phys. 75, 4765 (1981).

${ }^{14}$ A. Adams, J. Moreland, and P. K. Hansma, Surf. Sci. 111, 351 (1981)

${ }^{15}$ P. K. Aravind, E. Hood, and H. Metiu, Surf. Sci. 109, 95 (1981).

${ }^{16}$ A. Adams, J. Moreland, P. K. Hansma, and Z. Schlesinger, Phys. Rev. B 25, 3457 (1982).

${ }^{17}$ K. G. Sullivan, O. King, C. Sigg, and D. G. Hall, Appl. Opt. 33, 2447 (1994).

${ }^{18}$ J. M. Lupton, B. J. Matterson, I. D. W. Samuel, M. J. Jory, and W. L. Barnes, Appl. Phys. Lett. 77, 3340 (2000). sample are a possible indication of the variation in dipole sites above the grating surface.

\section{ACKNOWLEDGMENTS}

The authors thank Richard Amos and Philip Worthing for many fruitful discussions and extend their gratitude to DERA Malvern and the United Kingdom EPSRC for supporting this work.

${ }^{19}$ N. E. Hecker, R. A. Höpfel, N. Sawaki, T. Maier, and G. Strasser, Appl. Phys. Lett. 75, 1577 (1999).

${ }^{20}$ J. Moreland, A. Adams, and P. K. Hansma, Phys. Rev. B 25, 2297 (1982).

${ }^{21}$ P. T. Worthing and W. L. Barnes (unpublished).

${ }^{22}$ J. Arias, P. K. Aravind, and H. Metiu, Chem. Phys. Lett. 85, 404 (1981).

${ }^{23}$ P. T. Leung, Z. C. Wu, D. A. Jelski, and T. F. George, Phys. Rev. B 36, 1475 (1987); P. T. Leung and T. F. George, ibid. 36, 4664 (1987); P. T. Leung, Y. S. Kim, and T. F. George, ibid. 38, 10032 (1988); M. M. Hider and P. T. Leung, ibid. 44, 3262 (1991); W. L. Blacke and P. T. Leung, ibid. 56, 12625 (1997).

${ }^{24}$ H. Rigneault, F. Lemarchand, and A. Sentenac, J. Opt. Soc. Am. A 17, 1048 (2000).

${ }^{25}$ R. M. Amos and W. L. Barnes, Phys. Rev. B 59, 7708 (1999).

${ }^{26}$ J. Chandezon, M. T. Dupuis, G. Cornet, and D. Maystre, J. Opt. Soc. Am. 72, 839 (1982).

${ }^{27}$ E. L. Wood, J. R. Sambles, N. P. Cotter, and S. C. Kitson, J. Mod. Opt. 42, 1343 (1995).

${ }^{28}$ M. G. Salt, P. Andrew, and W. L. Barnes, J. Opt. Soc. Am. B 18, 240 (2001).

${ }^{29}$ I. V. Langmuir and V. J. Schaefer, J. Am. Chem. Soc. 60, 1351 (1938).

${ }^{30}$ R. G. Greenler, Surf. Sci. 69, 647 (1977).

${ }^{31}$ W. L. Barnes and J. R. Sambles, Surf. Sci. 177, 399 (1986).

${ }^{32}$ P. Andrew, Ph.D. thesis, University of Exeter, 1998.

${ }^{33}$ A. P. Alivisatos, M. F. Arndt, S. Efrima, D. H. Waldeck, and C. B. Harris, J. Chem. Phys. 86, 6540 (1987).

${ }^{34}$ D. Maystre, M. Nevière, and R. Petit, in Electromagnetic Theory of Gratings, edited by R. Petit (Springer-Verlag, Berlin, 1980), Chap. 6, p. 184.

${ }^{35}$ I. Pockrand, Phys. Lett. 49A, 249 (1974).

${ }^{36}$ W. L. Barnes, S. C. Kitson, T. W. Preist, and J. R. Sambles, J. Opt. Soc. Am. A 14, 1654 (1997).

${ }^{37}$ P. Andrew (unpublished).

${ }^{38}$ R. X. Bian, R. C. Dunn, X. S. Xie, and P. T. Leung, Phys. Rev. Lett. 75, 4772 (1995).

${ }^{39}$ H. Gersen, M. F. García-Parajó, L. Novotny, J. A. Veerman, L. Kuipers, and N. F. van Hulst, Phys. Rev. Lett. 85, 5312 (2000). 